\title{
PPAR $\gamma$ signaling is required for mediating EETs protective effects in neonatal cardiomyocytes exposed to LPS
}

\author{
Victor Samokhvalov' ${ }^{1}$, Jelle Vriend ${ }^{1,2}$, Kristi L. Jamieson ${ }^{1}$, Maria K. Akhnokh ${ }^{1}$, Rajkumar Manne $^{3}$, \\ John R. Falck ${ }^{3}$ and John M. Seubert ${ }^{1,4}$ * \\ ' Faculty of Pharmacy and Pharmaceutical Sciences, University of Alberta, Edmonton, AB, Canada \\ 2 Department of Chemistry and Pharmaceutical Sciences, Faculty of Sciences, VU University, Amsterdam, Netherlands \\ ${ }^{3}$ Department of Biochemistry and Pharmacology, University of Texas Southwestern Medical Center, Dallas, TX, USA \\ ${ }^{4}$ Department of Pharmacology, Faculty of Medicine and Dentistry, University of Alberta, Edmonton, AB, Canada
}

\section{Edited by:}

Chiranjib Chakraborty, Galgotias

University, India

\section{Reviewed by:}

Rodrigo Guabiraba, French National Institute for Agricultural Research,

France

Md Abdul Hye Khan, Medical College of Wisconsin, USA

\section{*Correspondence:}

John M. Seubert, Faculty of Pharmacy and Pharmaceutical Sciences, University of Alberta, 2020-M Katz Group Centre for Pharmacy and Health Research, 11361-87 Avenue, Edmonton, AB T6G 2E1, Canada e-mail: jseubert@ualberta.ca
Lipopolysaccharide (LPS) is a bacterial wall endotoxin producing many pathophysiological conditions including myocardial inflammation leading to cardiotoxicity. Epoxyeicosatrienoic acids (EETs) are biologically active metabolites of arachidonic acids capable of activating protective cellular pathways in response to stress stimuli. EETs evoke a plethora of pathways limiting impairments of cellular structures, reducing cell death, and promoting anti-inflammatory reactions in various cell types. Considering EETs are capable of producing various biological protective effects, we hypothesized that EETs would protect rat neonatal cardiomyocytes (NCM) against LPS-induced cytotoxicity. In this study, we used a dualacting, synthetic analog of EETs, UA-8 [13-(3-propylureido)tridec-8-enoic acid], possessing both EET-mimetic and soluble epoxide hydrolase selective inhibitory properties and 14,15EET as a model of canonical EET molecules. We found that both UA-8 and 14,15-EET significantly improved cell viability and mitochondrial function of cardiomyocytes exposed to LPS. Furthermore, treatment with UA-8 or 14,15-EET resulted in significant attenuation of LPS-triggered pro-inflammatory response, caspase-3 activation and reduction in the total antioxidant capacity in cardiomyocytes. Importantly, EET-mediated effects were significantly reduced by pharmacological inhibition of peroxisome proliferator-activated receptors $\gamma$ (PPAR $\gamma$ ) suggesting that PPAR $\gamma$ signaling was required for EETs exerted protective effects. Data presented in the current study demonstrate that activation of PPAR $\gamma$ signaling plays a crucial role in EET-mediated protection against LPS-cytotoxicity in cardiomyocytes.

Keywords: epoxyeicosatrienoic acid, cardiac cells, LPS, inflammation, PPAR $\gamma$

\section{INTRODUCTION}

Inflammation is a complex and highly orchestrated process involved in protecting cells from injury; yet, mounting evidence suggests that overactivated inflammatory responses contribute to the initiation and development of a wide range of diseases including cardiovascular diseases (CVDs) (Gabay and Kushner, 1999; Ridker and Silvertown, 2008). Bacterial endotoxin lipopolysaccharide (LPS) is considered one of the major causes in initiating low-grade systemic inflammation associated with cardiac dysfunction (Niebauer et al., 1999; Opal et al., 1999). Binding of LPS to TLR-4 receptors results in execution of the IKK-NF-kB inflammatory program (Akira etal., 2001) leading to the release of pro-inflammatory cytokines such as TNF $\alpha$, IL-6, IL-1, and MCP-1 (Ohlsson et al., 1990; Carlson et al., 2005; Fallach et al., 2010). The LPS-triggered release of pro-inflammatory cytokines can directly cause cardiac damage via numerous mechanisms including activation of JNK signaling (Hambleton et al., 1996; Charalambous et al., 2007; Drosatos et al., 2011), increased oxidative stress (BenShaul etal., 2001), decreased $\beta$-adrenergic activity (Yasuda and Lew, 1997), reduced peroxisome proliferator-activated receptors (PPARs) expression and DNA binding activity (Feingold et al.,
2004; Maitra et al., 2009; Samokhvalov et al., 2012) or direct mitochondrial damage (Choumar et al., 2011). Although LPS triggered responses are well documented, the precise cellular and molecular mechanism(s) involved in LPS-induced myocardial dysfunction remains very poorly delineated.

Arachidonic acid is a 20 -carbon polyunsaturated fatty acid found in cell membranes. Activation of phospholipase A2 results in the release of arachidonic acid, which can undergo enzymatic conversion forming biologically active lipid molecules (Rosenthal et al., 1995; Roman, 2002; Levick et al., 2007). Cytochrome P450 (CYP) epoxygenases are known to metabolize arachidonic acid into four regioisomeric epoxide metabolites, epoxyeicosatrienoic acids (EETs): 5,6-, 8,9-, 11,12-, and 14,15-EET (Fang etal., 2001; Kim et al., 2004). Reported EET-mediated effects include enhanced autophagy (Samokhvalov etal., 2013), inhibition of apoptosis (Dhanasekaran et al., 2008), mitochondrial protection (Katragadda etal., 2009) and cell proliferation (Imig, 2012). Numerous in vitro and in vivo studies provide strong evidence that EETs have anti-inflammatory properties (Node et al., 1999; Deng et al., 2010; Imig, 2012), which involves inhibition of the IKKNF-kB cascade (Rompe et al., 2010). For example, 11,12-EET was 
found to prevent LPS-triggered activation of the inflammatory response in monocytes by suppressing NF-kB signaling (Kozak etal., 2003). However, the exact role EETs have in regulating anti-inflammatory reactions in cardiac cells remains unknown.

Preventing a pathological activation of the inflammatory response requires a tight coordination of biological processes directed to effectively suppress the pro-inflammatory response while promoting anti-inflammatory reactions (Chinetti et al., 2001; Jones etal., 2002; Liu et al., 2005; Moraes et al., 2006). PPARs are ligand-activated transcription factors and members of the nuclear hormone receptor superfamily (Wray and BishopBailey, 2008). PPAR nuclear receptors sense various biological molecules and regulate many cellular functions such as fatty acid metabolism and lipid transport (Desvergne and Wahli, 1999), inflammatory responses (Wang et al., 2002; Moraes et al., 2006), cell differentiation (Barak et al., 1999) and tissue development (Rosen etal., 1999). There are three PPAR isoforms characterized $(\alpha, \gamma$, and $\beta / \delta)$ that regulate physiologically distinct processes (Delerive et al., 2001; Bocher et al., 2002). Importantly, activation of PPARs, particularly PPAR $\gamma$, suppresses NF-kB-induced expression of inflammatory cytokines (Liu et al., 2005; Wang et al., 2010). Interestingly, EETs have been identified as potent PPARs activators (Node etal., 1999; Liu et al., 2005; Ng et al., 2007), suggesting that anti-inflammatory effects of EETs might be mediated via PPAR-signaling. Despite already published studies, the existing knowledge regarding the mechanisms through which EETs attenuate LPS-induced cytotoxicity appears to be insufficient. Considering LPS down-regulates PPAR-mediated signaling, thus initiating the pro-inflammatory response, our objective was to determine if the anti-inflammatory effects of EETs required activation of $\operatorname{PPAR} \gamma$ signaling in neonatal cardiomyocytes (NCMs).

\section{MATERIALS AND METHODS CELL CULTURE}

Neonatal cardiomyocytes were isolated from 3 day-old pups as described before (Samokhvalov et al., 2012). Each isolation was done on a different day to perform a separate set of experiments $(N=3-4)$. Isolated NCMs were cultivated in DMEM medium supplemented with $10 \%$ FBS at $37^{\circ} \mathrm{C}$ in a humidified incubator maintaining $5 \% \mathrm{CO}_{2}$ and $95 \%$ air. Cell viability was assessed using a Trypan Blue exclusion assay as previously described (Samokhvalov etal., 2013). Beating rate of cardiomyocytes was evaluated by counting the number of beats per min in five different cell clusters in five independently blinded experiments.

\section{TREATMENT PROTOCOLS}

In this study, NCMs were treated with LPS $(1 \mu \mathrm{g} / \mathrm{ml})$, a novel EETanalog, UA-8 [13-(3-propylureido)tridec-8-enoic acid $(1 \mu \mathrm{M})$ ], that possesses EET-mimetic and soluble epoxide hydrolase (sEH) inhibitory properties, and/or 14,15-EET $(1 \mu \mathrm{M})$ as a model EETs (Batchu et al., 2011). The chemical structure and properties of the UA-8 were previously described and depicted in Figure 1A; UA-8 can inhibit sEH at nanomolar concentrations $\left(\mathrm{IC}_{50} 46 \mathrm{nM}\right.$; Batchu et al., 2011). In order to block EET-mediated effects, we utilized the antagonist, 14,15-epoxyeicosa-5( $Z$ )-enoic acid (14,15-EEZE, $10 \mu \mathrm{M}) . \operatorname{PPAR} \gamma$ was inhibited with a specific pharmacological agent GW9662 ( $1 \mu \mathrm{M}$; Seargent et al., 2004). Stock solutions of UA-8 and GW9662 were prepared in DMSO while 14,15-EET, and 14,15-EEZE were prepared in 100\% ethanol, final concentrations of both solvents were less than $0.01 \%$ of the treatment solutions.

\section{METABOLIC ASSESSMENTS}

In order to test overall efficiency of mitochondrial oxidative metabolism, we used a kit (Sigma-Aldrich, Co., Oakville, ON, CAN) measuring ADP/ATP ratio in cell lysates by luciferase-based method. The intensity of emitted light occurred during the first reaction is proportional to the level of ADP in the sample while the intensity of the second reaction reflects the level of ATP. Alternatively, MTT assay was employed to examine total oxidative metabolism as previously described (Samokhvalov et al., 2013). The intensity of reduction of 3-(4,5-dimethylthiazol-2-yl)-2,5diphenyltetrazolium bromide to formazan crystals by mitochondrial dehydrogenases positively correlates with the overall activity of oxidative metabolism (Wang et al., 2010). Optical density of DMSO extracted formazan was measured spectrophotometrically at $595 \mathrm{~nm}$.

\section{TOTAL ANTIOXIDANT CAPACITY, CASPASE-3, AND 20S PROTEASOME ACTIVITY ASSAYS}

We determined the total antioxidant activity to provide an indication of the relative ROS status. Briefly, the principle of the antioxidant assay is formation of a ferryl myoglobin radical from metmyoglobin and hydrogen peroxide, which oxidizes the ABTS [2,2'-azino-bis(3-ethylbenzothiazoline-6-sulfonic acid) producing a radical cation, ABTS+, a soluble chromogen that is green in color and can be determined spectrophotometrically at $405 \mathrm{~nm}$. (Sigma-Aldrich, Co, Oakville, ON, USA). To assess activation of apoptosis, we measured caspase-3 activity by employing a spectrofluorimetric assay, which detects AMC fluorescence after cleavage of AC-DEVD-AMC substrate as described previously (Seubert et al., 2002). Total proteasome activity as a marker of unspecific degenerative processes (Samokhvalov et al., 2013) was determined in the whole cell lysates based on monitoring the release of AMC by proteolytic cleavage of the peptide Suc-LLVYAMC (CHEMICON Inc, Billerica, MA, USA) by 20 S proteasomes. Fluorescence was monitored in both caspase- 3 and total proteasome assays at wavelengths of $380 \mathrm{~nm}$ (excitation) and $460 \mathrm{~nm}$ (emission). Specific activities were determined from a standard curve established with AMC.

\section{CYTOKINES ASSAY}

Medium was centrifuged ( $5 \mathrm{~min}$ at $5000 \mathrm{~g}$ ) supernatants were analyzed by ELISA for rat TNF $\alpha$ and MCP-1 (ABCAM, Cambridge, $\mathrm{UK})$.

\section{NF-kB AND PPAR $\gamma$ DNA BINDING ASSAYS}

NF-kB DNA binding assay was measured using an ELISA kit from Active Motif (Carlsbad, CA, USA). PPAR $\gamma$ DNA biding activity was measured using an ELISA kit from ABCAM (Cambridge, UK). Briefly, the assays are based on the specific recognition of PPAR $\gamma$ or NF-kB response elements by intracellular PPAR $\gamma$ or NF-kB transcription factors contained in cell lysates. 
A<smiles>NC(=O)CCCCCCCCOC1CO1</smiles>

14,15-EET

B

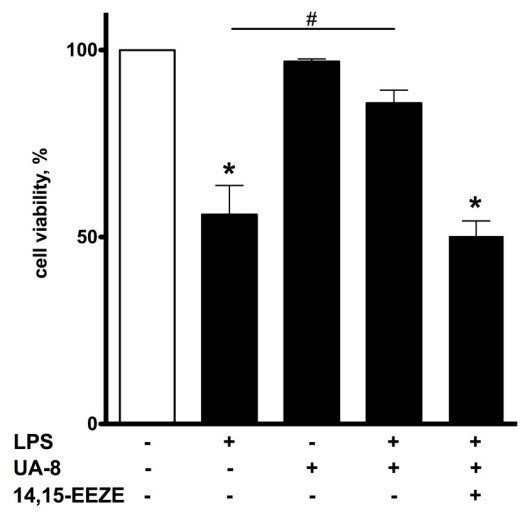

D

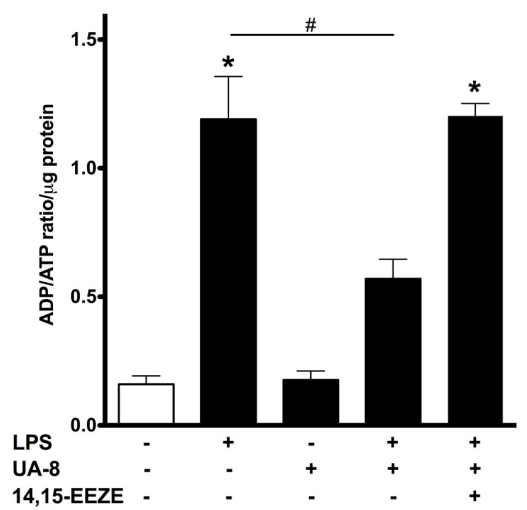

FIGURE 1 | Treatment with UA-8 attenuates LPS-induced decrease in cell viability, oxidative metabolic activity and improves contractility of neonatal cardiomyocytes. NCMs were treated with LPS $(1 \mu \mathrm{g} / \mathrm{ml})$ and/or UA-8 $(1 \mu \mathrm{m})$ in the presence or absence of 14,15-EEZE $(10 \mu \mathrm{m})$ for 24 h. (A) Chemical structures of 14,15-EET, UA-8, 14,15-EEZE.

(B) UA-8 improved LPS-lowered cell viability. (C) Mitochondrial oxidative

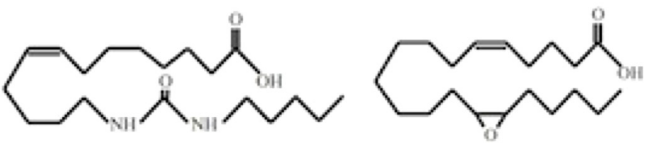

UA-8

14,15-EEZE

C

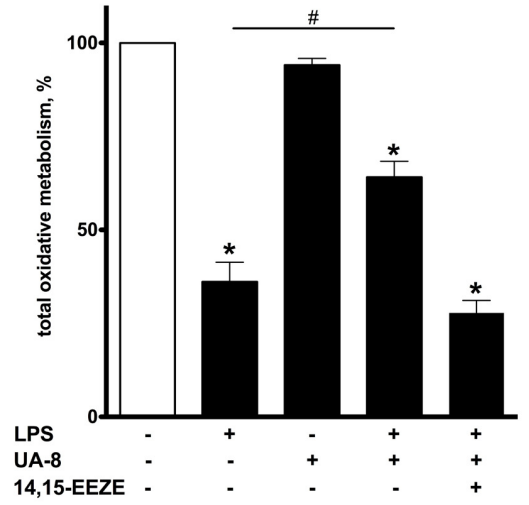

E

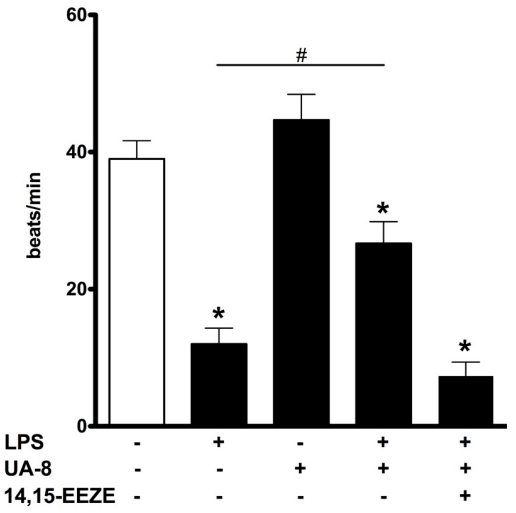

metabolic activity was preserved by treatment with UA-8. (D) UA-8 prevented LPS-induced increase in ADP/ATP ratio. (E) UA-8 attenuated LPS-impaired contractility of NCMs. Values are represented as mean \pm SEM,$N=3-4$. Significance was set at $P<0.05$.

* Significantly different from control. \# Significantly different from LPS-treated cells.

\section{STATISTICAL ANALYSIS}

Data are presented as mean \pm SEM. Statistical analysis was based on one-way ANOVA with a Bonferonni post hoc test; $P<0.05$ was considered statistically significant.

\section{RESULTS}

TREATMENT WITH UA-8 ATTENUATED LPS-INDUCED DECREASE IN CELL VIABILITY AND FUNCTIONAL ACTIVITY

Lipopolysaccharide is a well-known cytotoxic agent promoting rapid cell death (Charalambous et al., 2007). After $24 \mathrm{~h}$, more than $50 \%$ of NCMs exposed to LPS were dead as evaluated by a Trypan blue exclusion assay (Figure 1B). Addition of UA-8 dramatically prevented LPS-triggered decrease in cell viability. However, co-treatment with 14,15-EEZE, an EET antagonist, abolished UA-8 protection against LPS (Figure 1B). In order to further examine protective effect of UA-8, we examined total oxidative metabolism in cardiomyocytes exposed to LPS by MTT assay. Figure 1C demonstrates that treatment with LPS for $24 \mathrm{~h}$ caused a significant decline in oxidative metabolic activity suggesting that mitochondrial function was severely compromised. Treatment with UA-8 ameliorated LPS-induced exacerbation in mitochondrial function, which was in turn abolished by a co-treatment with 14,15-EEZE. Paralleling our observations with regard to impairments in mitochondrial function, LPS also caused a robust 
increase in ADP/ATP ratio that was greatly reversed by addition of UA-8 (Figure 1D), suggesting mitochondria in LPS treated NCMs could no longer meet cellular demands for ATP. The ability of cardiomyocytes to contract in vitro reflects their functional activity and requires a continuous supply of ATP to sustain normal contractility (Yasuda and Lew, 1997). We found that treatment with LPS for $24 \mathrm{~h}$ induced a dramatic reduction in contractile activity of NCMs indicative of severe impairments in their functional activity (Figure 1E). Treatment with UA-8 prevented the loss of contractile activity in NCMs, which was abolished by co-treatment with 14,15-EEZE. These data demonstrate that the LPS triggered mitochondrial dysfunction in NCMs led to ATP deprivation associated with decreased contractility and resulting in cell death, was rescued by treatment with UA- 8 .

\section{LPS-INDUCED ACTIVATION OF INFLAMMATORY RESPONSE IS ATTENUATED BY UA-8}

Lipopolysaccharide-induced cytotoxicity is largely mediated through robust activation of the pro-inflammatory response (Charalambous etal., 2007). Therefore, it was important to investigate if inflammatory markers were up-regulated in our experimental model of LPS-induced cytotoxicity and whether treatment with UA-8 could reduce them. Accordingly, we first assessed NF-kB DNA binding activity, a test revealing functional activity of the major factor orchestrating the pro-inflammatory response. Figure 2A illustrates that treatment with LPS for $24 \mathrm{~h}$ induced a pronounced increase in NF-kB DNA binding activity. Furthermore, we also demonstrate that LPS promoted a strong release of the major pro-inflammatory cytokines TNF $\alpha$ and MCP-1 from cardiomyocytes (Figures 2B,C). These changes in the levels of the inflammatory markers indicate a profound activation of the pro-inflammatory response caused by LPS. Co-treatment of cells with UA-8 significantly attenuated the LPS-triggered inflammatory response in cardiomyocytes as seen with reduced NF-kB DNA binding activity and decreased release of TNF $\alpha$ and MCP-1 cytokines. Co-treatment with 14,15EEZE abolished the anti-inflammatory effects of UA-8, thus suggesting the UA- 8 associated improvements in LPS-triggered inflammatory response were realized through EET-specific pathways.

\section{UA-8 AMELIORATES CELLULAR STRESS REACTIONS IN RESPONSE TO LPS}

Oxidative stress has been recognized as a major unspecific stress reaction mediating LPS cytotoxicity (Ben-Shaul et al., 2001). In order to examine the involvement of oxidative stress in LPSinduced cytotoxicity, we employed a test measuring a total pool of enzymatic and non-enzymatic components of the cellular antioxidant defense, thereby revealing cell ability to withstand oxidative stress. We found that LPS caused a collapse in total antioxidant capacity of cardiomyocytes indicative of activated oxidative stress. However, treatment with UA-8 significantly preserved the total antioxidant capacity of LPS-exposed cardiomyocytes, thus, providing a piece of evidence that activation of oxidative stress did not occur in full (Figure 3A). The accumulation of ubiquinated proteins triggers $20 \mathrm{~S}$ proteasome activity to remove the targeted damaged proteins. As such, $20 \mathrm{~S}$ proteasome activity can be utilized as a marker of unspecific cellular degenerative processes (Samokhvalov et al., 2013). Interestingly, treatment with LPS failed to induce any alterations in 20 S proteasome activity (Figure 3B). LPS triggers a number of complex degenerative reactions culminating in cell death, often through apoptosis (Turdi et al., 2012). Consistent with these reports, Figure 3C demonstrates that treatment with LPS provoked a robust activation of caspase-3, which indicates initiation of apoptosis. Treatment with UA-8 dramatically decreased LPS-induced activation of caspase-3. Importantly, all observed effects of UA-8 were abolished by a co-treatment with 14,15-EEZE providing further support for the involvement of specific EET's signaling.

\section{PPAR $\gamma$ INHIBITION PREVENTS UA-8-ASSOCIATED PROTECTIVE EFFECTS IN LPS-INDUCED CYTOTOXICITY}

Peroxisome proliferator-activated receptors nuclear receptors are broadly recognized as signaling factors, which are involved in regulating inflammatory responses. Activation of PPARs can both stimulate an anti-inflammatory response and suppress the
$\mathbf{A}$

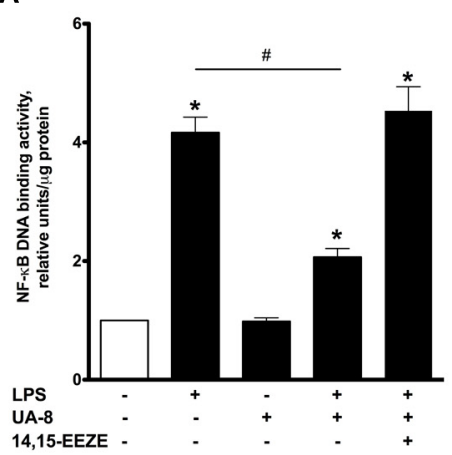

B

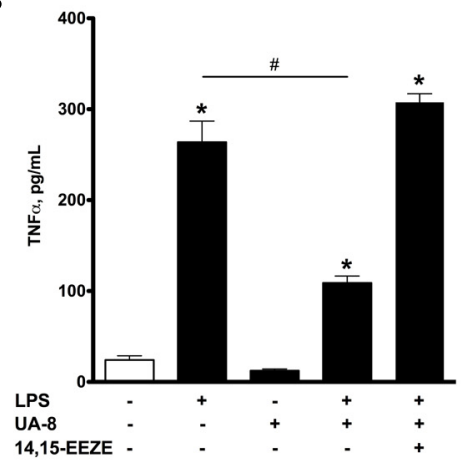

C

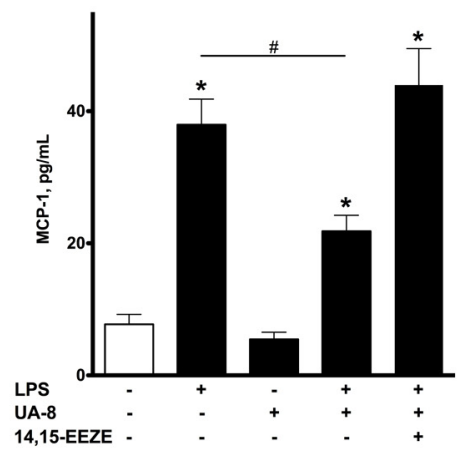

FIGURE 2 | UA-8 inhibited inflammatory response triggered by LPS in NCMs. NCMs were treated with LPS $(1 \mu \mathrm{g} / \mathrm{ml})$ and/or UA-8 $(1 \mu \mathrm{m})$ in the presence or absence of 14,15-EEZE $(10 \mu \mathrm{m})$ for $24 \mathrm{~h}$. (A) UA-8 suppressed LPS-triggered up-regulation of NF-kB DNA binding activity in
NCMs. (B) and (C) UA-8 robustly diminished the release of TNF $\alpha$ and MCP-1 from NCMs exposed to LPS. Values are represented as mean \pm SEM, $N=3-4$. Significance was set at $P<0.05$. * Significantly different from control. \# Significantly different from LPS-treated cells. 
A

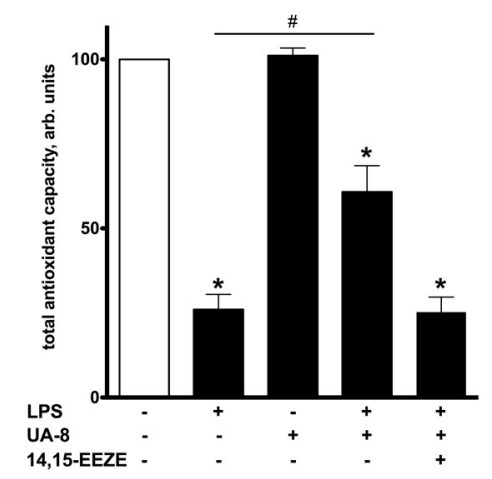

B

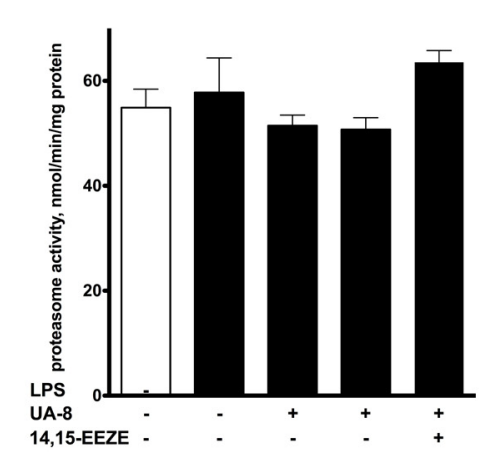

C

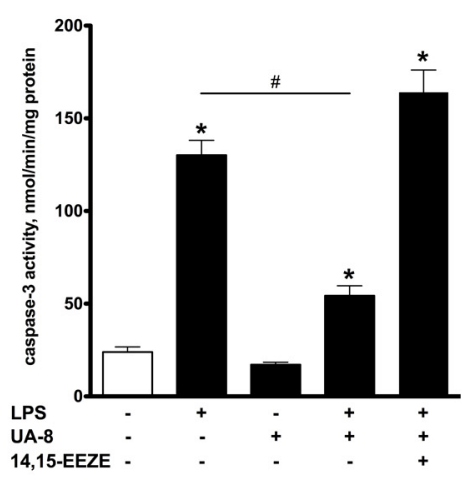

FIGURE 3 | Treatment with UA-8 reduced LPS-evoked activation of cellular stress responses. NCMs were treated with LPS $(1 \mu \mathrm{g} / \mathrm{ml})$ and/or UA-8 $(1 \mu \mathrm{m})$ in the presence or absence of 14,15-EEZE $(10 \mu \mathrm{m})$ for $24 \mathrm{~h}$. (A) UA-8 sustained the total antioxidant capacity of NCMs exposed to LPS. (B) No changes were observed in $20 \mathrm{~S}$ total proteasome activity after treatment either with UA-8 or with LPS (C) UA-8 prevented LPS-induced activation of caspase-3. Values are represented as mean $\pm \mathrm{SEM}, N=3-4$. Significance was set at $P<0.05$. * Significantly different from control. \# Significantly different from LPS-treated cells. pro-inflammatory response. While EETs activate PPARs through ligand-specific interaction (Liu et al., 2005; Ng et al., 2007), LPS causes a reduction in the expression of PPARs (Feingold et al., 2004; Maitra et al., 2009). Thus, we explored whether UA-8 associated protective effects occurred through PPAR-signaling. First, we assessed if treatments with UA-8 and/or LPS could affect PPAR $\gamma$ DNA binding activity. We found that treatment with LPS caused a dramatic reduction in PPAR $\gamma$ DNA binding activity. In contrast, treatment with UA- 8 strongly enhanced PPAR $\gamma$ DNA binding activity. Furthermore, treatment with UA-8 also significantly restored LPS-induced drop in PPAR $\gamma$ DNA binding activity (Figure 4). In order to examine the role of PPAR $\gamma$, we treated cardiomyocytes with GW 9662, a specific pharmacological inhibitor of PPAR $\gamma$ signaling. Pharmacological inhibition of PPAR $\gamma$ with GW $9662(1 \mu \mathrm{M})$ was confirmed by assessing DNA binding activity, which showed strongly reduced PPAR $\gamma$ DNA binding in all experimental groups (Figure 4). Pharmacological inhibition of PPAR $\gamma$ prevented UA-8-associated protective effects. Our data demonstrate that treatment with GW 9662 blocked UA-8 improved cell viability (Figure 5A) and ADP/ATP ratio (Figure 5B) in LPS-treated NCMs. Furthermore, pharmacological inhibition of PPAR $\gamma$ with GW 9662 prevented UA-8-evoked anti-inflammatory and anti-apoptotic effects as observed with NFkB DNA binding (Figure 5C) and caspase-3 activities (Figure 5D) in NCMs exposed to LPS. These results provide evidence that UA-8-associated protective effects require activation of PPAR $\gamma$ signaling.

\section{4,15-EET RECAPITULATES PROTECTIVE EFFECTS ASSOCIATED WITH UA-8}

UA-8 represents a synthetic compound with structural similarities to EETs and sEH inhibitor properties (Batchu et al., 2011). Thus, in order to further determine if the effects were mediated by EET-mediated events, we utilized 14,15-EET as a model to explore similarities observed with UA-8. NCMs treated with 14,15-EET $(1 \mu \mathrm{M})$ demonstrated significantly better cell viability following

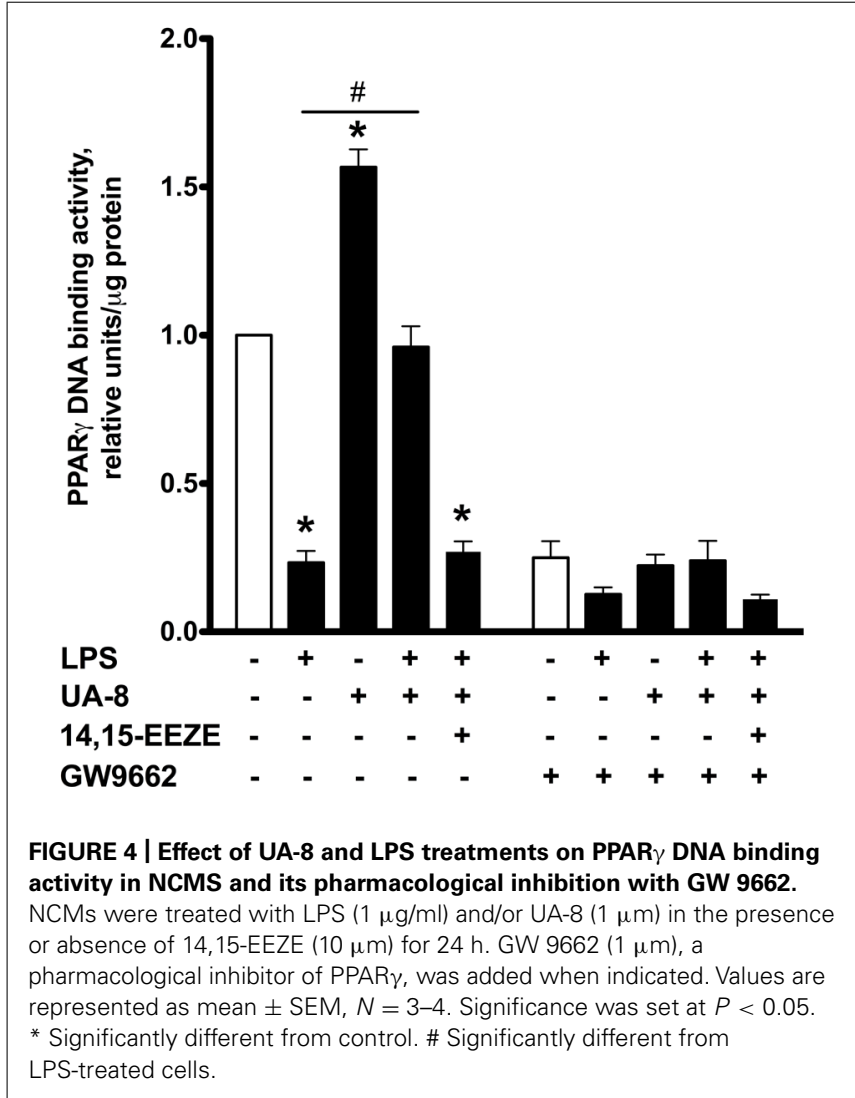

LPS treatment (Figure 6A). Furthermore, treatment with 14,15EET significantly reduced LPS-triggered NF-kB DNA binding activity and release of TNF $\alpha$ (Figures 6B,C). These observations illustrate that treatment with 14,15-EET effectively suppressed LPS-induced pro-inflammatory responses in NCMs. Finally, treatment with 14,15-EET significantly enhanced PPAR $\gamma$ DNA binding activity while limited LPS-induced drop in PPAR $\gamma$ DNA binding 
A

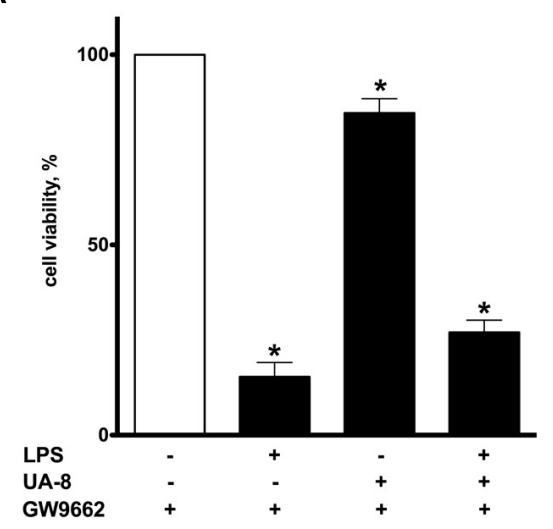

C

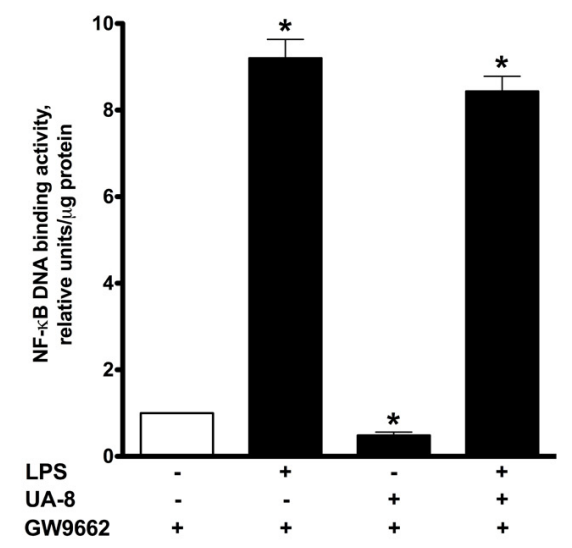

FIGURE 5 | Pharmacological inhibition of PPAR $\gamma$ significantly prevented UA-8 associated protective effects in LPS-induced cytotoxicity. NCMs were treated with LPS $(1 \mu \mathrm{g} / \mathrm{ml})$ and/or UA-8 $(1 \mu \mathrm{m})$ in the presence of GW $9662(1 \mu \mathrm{m})$ for $24 \mathrm{~h}$. (A) Pharmacological inhibition of PPAR prevented protective effect of UA-8 on cell viability. (B) Inhibition of PPAR $\gamma$ abolished protective effect of UA-8 on ADP/ATP ratio in NCMS
B

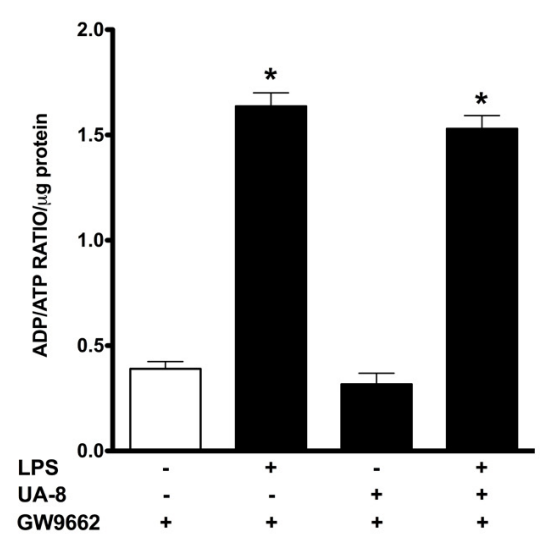

D

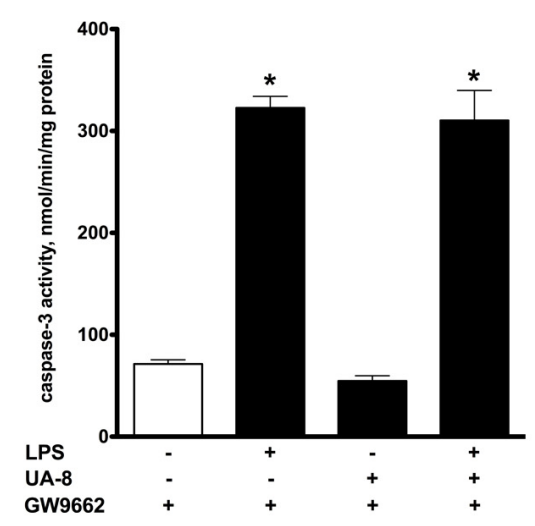

exposed to LPS. (C) UA-8 did not reduce LPS-enhanced NF-kB DNA binding activity in NCMs treated with the pharmacological inhibitor of PPAR $\gamma$. (D) Inhibition of PPAR $\gamma$ limited UA-8 inhibitory effect on caspase-3 activity in NCMs exposed to LPS. Values are represented as mean $\pm \operatorname{SEM}, N=3-4$. Significance was set at $P<0.05$. * Significantly different from control. activity in NCMs (Figure 6D). Importantly, addition of 14,15EEZE abolished all protective effects of 14,15-EET similarly as it was observed with UA-8. Thus, protective effects of UA-8 and 14,15-EET in LPS-induced cytotoxicity were biologically very similar and sensitive to 14,15-EEZE suggesting they occurred through an eicosanoid-specific signaling pathway(s).

\section{DISCUSSION}

In the present study, we provide evidence that EET-mediated activation of PPAR $\gamma$ signaling is required to reduce LPS-induced cytotoxicity in cardiomyocytes, demonstrating EETs may directly regulate cardiac inflammatory responses.

Myocardial exposure to LPS can trigger low-grade inflammation reactions, which are initiated through NF- $\kappa$ B signaling leading to release of cytokines such as TNF $\alpha$ and MCP-1 (Charalambous et al., 2007). The subsequent stress and inflammatory response triggers a cascade of events that may cause deleterious alterations in mitochondrial function, leading to apoptotic cell death (Oddis and Finkel, 1995; Ben-Shaul et al., 2001; Choumar et al., 2011). For example, LPS treatment has been reported to induce depletion of cardiac ATP, which was associated with myocardium dysfunction (Drosatos et al., 2011). Consistent with the literature, our data demonstrated that LPS induced a rapid loss of cell viability and reduced mitochondrial oxidative metabolism. As expected, LPS triggered a robust activation of the pro-inflammatory response in cardiomyocytes as was seen with up-regulation of NF-kB DNA binding activity and a dramatic reduction in PPAR $\gamma$ DNA binding activity, which were followed by the release of TNF $\alpha$ and MCP-1. The LPS-instigated execution of the pro-inflammatory response was potentially the primary event causing activation of caspase- 3 and collapse of the antioxidant capacity in NCMs.

In this study, we demonstrate that EETs stimulate a number of adaptive responses enabling NCMs to withstand LPS-induced cytotoxicity. Particularly, our data highlight that treatment with EETs preserved cell viability, oxidative metabolic activity, diminished the pro-inflammatory response and reduced caspase- 3 activity in cardiomyocytes exposed to LPS. The protective effects of EETs were abolished by a co-treatment with its specific antagonist 
A

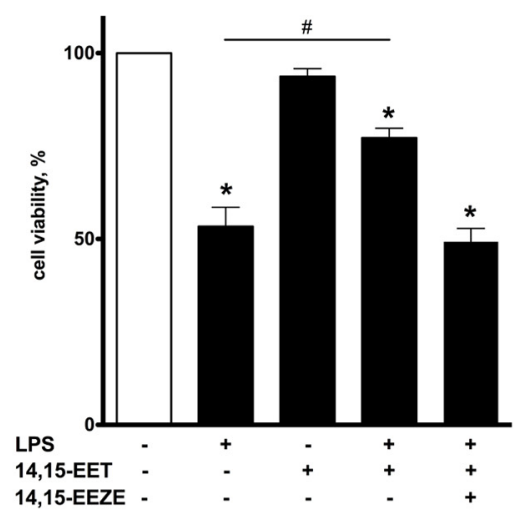

C

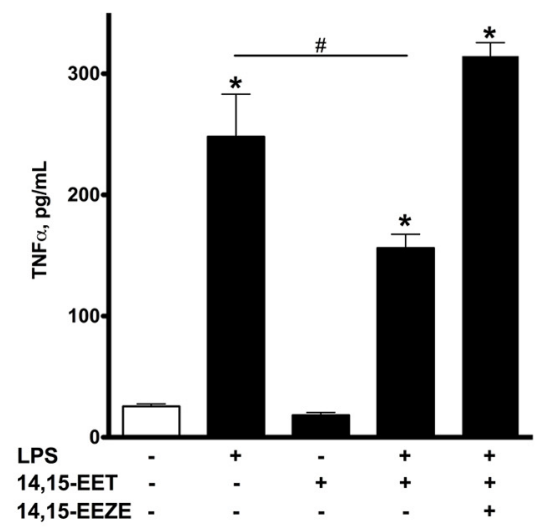

FIGURE 6 | Treatment with 14,15-epoxyeicosatrienoic acids (EET) recapitulated protective effects of UA-8 in LPS-induced cytotoxicity. NCMs were treated with LPS $(1 \mu \mathrm{g} / \mathrm{ml})$ and/or $14,15-$ EET $(1 \mu \mathrm{m})$ in the presence or absence of 14,15-EEZE $(10 \mu \mathrm{m})$ for $24 \mathrm{~h}$. (A) Treatment with 14,15-EET significantly improved LPS-decreased cell viability. (B,C) 14,15-EET significantly attenuated LPS-triggered inflammatory response
B

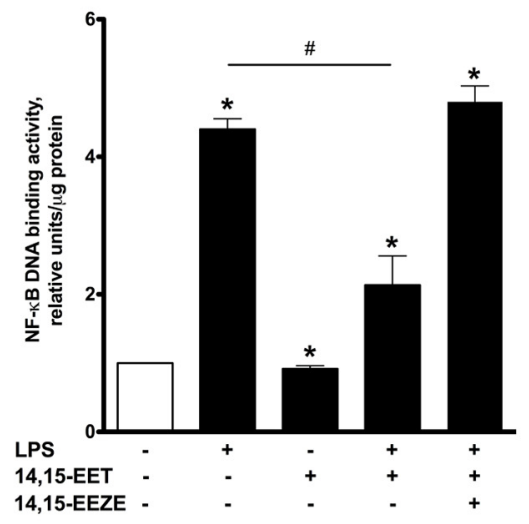

D

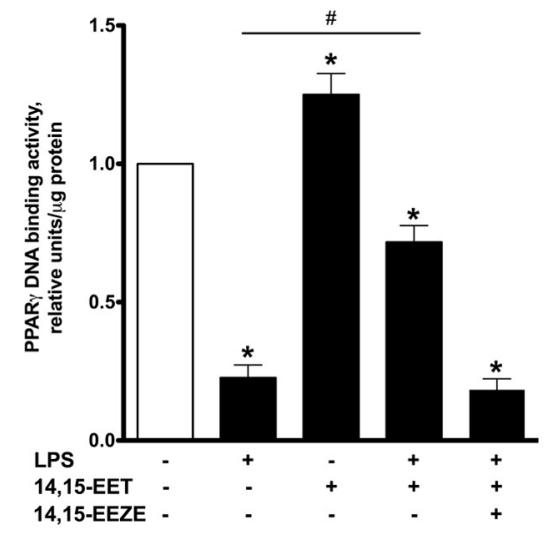

as seen with reductions in NF-kB DNA binding activity and TNF $\alpha$ release. (D) 14,15-EET induced an increase in PPAR $\gamma$ DNA binding activity and significantly prevented LPS-triggered drop in PPAR $\gamma$ DNA binding activity. Values are represented as mean \pm SEM, $N=3-4$. Significance was set at $P<0.05$. * Significantly different from control. \# Significantly different from LPS-treated cells.
14,15-EEZE providing evidence that the observed effects were attributed to EETs specific pathways. There are two key results that suggest the effects observed in the current study are attributed to EETs: (1) the biological response was completely attenuated by the EET-antagonist 14,15-EEZE; and, (2) we observe similar biological effects with 14,15-EET and UA-8. Thus, we can conclude the biological effects are mostly attributed to EET-mediated effects.

Although there is no EET specific receptor discovered yet, numerous studies show the ability of EETs to act as cellular signaling molecules regulating numerous pathways (Imig, 2012; Shahabi et al., 2014). Evidence suggests that EETs may act through a receptor(s) and demonstrate affinity to known receptors (Widstrom et al., 2003; Spector and Norris, 2007). Currently, there is limited information regarding the exact concentrations of EET regioisomers found in cardiomyocytes but evidence indicates bioavailability is influenced by various factors such as stress (Imig, 2012). Intriguingly, EETs may act as endogenous agonists to PPARs, which increases VEGF and angiogenesis in endothelial progenitor cells (Liu et al., 2005; Ng et al., 2007; Xu et al., 2013). In contrast, LPS has been shown to cause a dramatic reduction in the expression of PPARs (Feingold et al., 2004; Maitra et al., 2009). Previously, we reported that treatment with LPS resulted in a rapid decrease in PPAR $\alpha$ DNA binding activity in NCMs and PPAR $\gamma$ DNA binding activity in peritoneal macrophages. The primary observation from this study was that LPS-triggered a rapid decline in PPAR $\alpha$ and PPAR $\gamma$ DNA binding activities evoked a robust pro-inflammatory response in both NCMs and peritoneal macrophages. Furthermore, restoring PPAR $\alpha$ and PPAR $\gamma$ DNA binding activities by inhibiting malonyl-CoA decarboxylase significantly limited LPS-triggered inflammatory response (Samokhvalov etal., 2012). This may be a compelling observation validating the importance of finding novel agonists of PPARs to develop new strategies targeting LPS-induced cytotoxicity. Numerous reports postulate that activation of PPAR-signaling, particularly PPAR $\alpha$ and PPAR $\gamma$, can suppress the inflammatory response through inhibition of NF-kB pathways (Liu et al., 2005; 


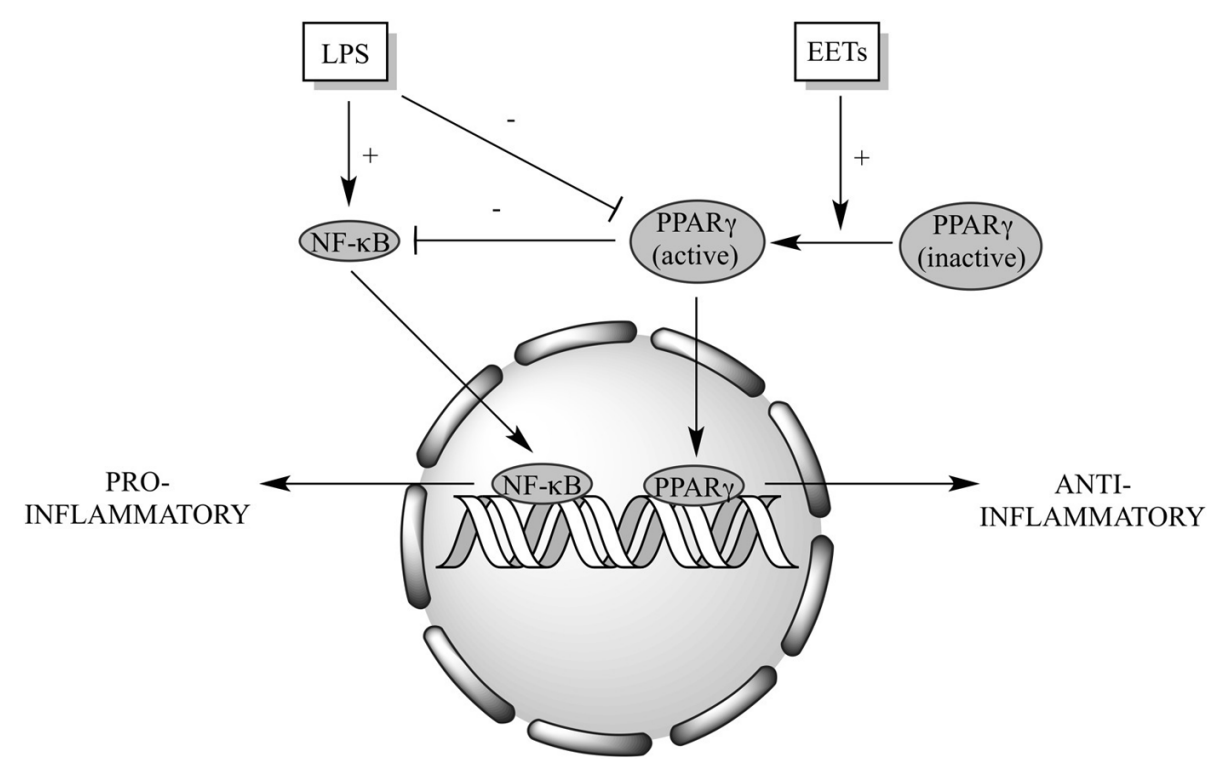

FIGURE 7 | Schematic of proposed EET cardioprotective mechanism(s). LPS triggers activation of NF-kB-dependent signaling and suppresses PPAR $\gamma$ activation, resulting in a shift of the cellular response to a pro-inflammatory state. Increased EETs can act as PPAR $\gamma$ agonists and inhibit NF-k B-dependent signaling, shifting the cellular response to an anti-inflammatory state.
Wray and Bishop-Bailey, 2008). Nonetheless, the protective effects of EETs are not limited to activating PPAR $\gamma$-mediated signaling pathways. There is strong evidence in the literature that EETs suppress NF-kB-mediated induction and the subsequent pro-inflammatory response through inhibition of IKK complex activity (Deng et al., 2010), which partially occurs via activation of PI3K-dependent Akt and EGF receptor signaling pathway (Zhao et al., 2012). The involvement of PI3K signaling in EET-mediated activation of PPAR $\gamma$ is possible mechanism of action. Our previously published studies suggest that EET triggered biologically effects might involve PI3K (Batchu et al., 2011, 2012). Evidence from the literature also suggests a complex interplay between PI3K and PPAR $\gamma$ pathways (Chuang et al., 2007; Mishra et al., 2010). Although these indirect pieces of evidence support the role of PI3K cascade in EET-mediated events, further evidence is required to understand the precise mechanism(s) of action. Considering evidence showing desensitization to LPS is favorable in patients with chronic heart failure (Charalambous et al., 2007), our current data imply targeting PPAR $\gamma$-mediated signaling with EETs would be a possible novel therapeutic approach to treat LPS-induced cardiac pathologies.

Enhanced release of pro-inflammatory cytokines from the heart causes recruitment of mononuclear cells which has been shown to have a role worsening the development of cardiovascular disease (Zernecke et al., 2008). The therapeutic potential for limiting inflammatory-induced migration of immune cells in myocardium appears to be tremendous. Our findings illustrate for the first time that EETs can effectively reduce LPStriggered release of the pro-inflammatory cytokines from NCMs. While not definitive, we can still tentatively suggest that acting as agonists to PPAR $\gamma$, EETs can suppress LPS-induced proinflammatory response thereby reducing cell death in NCMs.
Indeed, our observations indicate that activation of PPAR $\gamma$ signaling contribute significantly to EETs exerted protective effect. This notion was supported when EET-associated protective effects were abolished by pharmacological inhibition of PPAR $\gamma$ signaling.

In summary, we demonstrate a crucial role of PPAR $\gamma$ signaling in mediating EET protective effects toward LPS-induced cytotoxicity in cardiomyocytes (Figure 7). While the precise cellular and molecular mechanism(s) remain unknown, our data suggest EETs exert their protective effect through activation of antiinflammatory

processes.

\section{ACKNOWLEDGMENTS}

This work was supported by a grant from the National Science and Engineering Research Council (NSERC) of Canada (John M. Seubert). Jelle Vriend was supported by a grant from the Dutch Heart Foundation (2013SB007). John R. Falck was supported by the Robert A. Welch Foundation (I-0011) and NIH DK38226.

\section{REFERENCES}

Akira, S., Takeda, K., and Kaisho, T. (2001). Toll-like receptors: critical proteins linking innate and acquired immunity. Nat. Immunol. 2, 675-680. doi: $10.1038 / 90609$

Barak, Y., Nelson, M. C., Ong, E. S., Jones, Y. Z., Ruiz-Lozano, P., Chien, K. R., et al. (1999). PPAR gamma is required for placental, cardiac, and adipose tissue development. Mol. Cell. 4, 585-595. doi: 10.1016/S1097-2765(00)80209-9

Batchu, S. N., Chaudhary, K. R., El-Sikhry, H., Yang, W., Light, P. E., Oudit, G. Y., et al. (2012). Role of PI3Kalpha and sarcolemmal ATP-sensitive potassium channels in epoxyeicosatrienoic acid mediated cardioprotection. J. Mol. Cell. Cardiol. 53, 43-52. doi: 10.1016/j.yjmcc.2012.04.008

Batchu, S. N., Lee, S. B., Qadhi, R. S., Chaudhary, K. R., El-Sikhry, H., Kodela, R., et al. (2011). Cardioprotective effect of a dual acting epoxyeicosatrienoic acid analogue towards ischaemia reperfusion injury. Br. J. Pharmacol. 162, 897-907. doi: 10.1111/j.1476-5381.2010.01093.x 
Ben-Shaul, V., Lomnitski, L., Nyska, A., Zurovsky, Y., Bergman, M., and Grossman, S. (2001). The effect of natural antioxidants, NAO and apocynin, on oxidative stress in the rat heart following LPS challenge. Toxicol. Lett. 123, 1-10. doi: 10.1016/S0378-4274(01)00369-1

Bocher, V., Chinetti, G., Fruchart, J. C., and Staels, B. (2002). [Role of the peroxisome proliferator-activated receptors (PPARS) in the regulation of lipids and inflammation control]. J. Soc. Biol. 196, 47-52. doi: 10.1155/S111072430 4308065

Carlson, D. L., Willis, M. S., White, D. J., Horton, J. W., and Giroir, B. P. (2005). Tumor necrosis factor-alpha-induced caspase activation mediates endotoxin-related cardiac dysfunction. Crit. Care Med. 33, 1021-1028. doi: 10.1097/01.CCM.0000163398.79679.66

Charalambous, B. M., Stephens, R. C., Feavers, I. M., and Montgomery, H. E. (2007) Role of bacterial endotoxin in chronic heart failure: the gut of the matter. Shock 28, 15-23. doi: 10.1097/shk.0b013e318033ebc5

Chinetti, G., Fruchart, J. C., and Staels, B. (2001). Peroxisome proliferator-activated receptors (PPARs): nuclear receptors with functions in the vascular wall. $Z$. Kardiol. 90(Suppl. 3), 125-132. doi: 10.1007/s003920170034

Choumar, A., Tarhuni, A., Letteron, P., Reyl-Desmars, F., Dauhoo, N., Damasse, J., etal. (2011). Lipopolysaccharide-induced mitochondrial DNA depletion. Antioxid. Redox. Signal. 15, 2837-2854. doi: 10.1089/ars.2010.3713

Chuang, C. C., Yang, R. S., Tsai, K. S., Ho, F. M., and Liu, S. H. (2007). Hyperglycemia enhances adipogenic induction of lipid accumulation: involvement of extracellular signal-regulated protein kinase 1/2, phosphoinositide 3-kinase/Akt, and peroxisome proliferator-activated receptor gamma signaling. Endocrinology 148, 4267-4275. doi: 10.1210/en.2007-2179

Delerive, P., Fruchart, J. C., and Staels, B. (2001). Peroxisome proliferatoractivated receptors in inflammation control. J. Endocrinol. 169, 453-459. doi 10.1677/joe.0.1690453

Deng, Y., Theken, K. N., and Lee, C. R. (2010). Cytochrome P450 epoxygenases, soluble epoxide hydrolase, and the regulation of cardiovascular inflammation. J. Mol. Cell. Cardiol. 48, 331-341. doi: 10.1016/j.yjmcc.2009.10.022

Desvergne, B., and Wahli, W. (1999). Peroxisome proliferator-activated receptors: nuclear control of metabolism. Endocr. Rev. 20, 649-688. doi: 10.1210/edrv.20.5.0380

Dhanasekaran, A., Gruenloh, S. K., Buonaccorsi, J. N., Zhang, R., Gross, G. J., Falck, J. R., et al. (2008). Multiple antiapoptotic targets of the PI3K/Akt survival pathway are activated by epoxyeicosatrienoic acids to protect cardiomyocytes from hypoxia/anoxia. Am. J. Physiol. Heart Circ. Physiol. 294, H724-H735. doi: 10.1152/ajpheart.00979.2007

Drosatos, K., Drosatos-Tampakaki, Z., Khan, R., Homma, S., Schulze, P. C., Zannis, V. I., et al. (2011). Inhibition of c-Jun-N-terminal kinase increases cardiac peroxisome proliferator-activated receptor alpha expression and fatty acid oxidation and prevents lipopolysaccharide-induced heart dysfunction. J. Biol. Chem. 286, 36331-36339. doi: 10.1074/jbc.M111.272146

Fallach, R., Shainberg, A., Avlas, O., Fainblut, M., Chepurko, Y., Porat, E., et al. (2010). Cardiomyocyte Toll-like receptor 4 is involved in heart dysfunction following septic shock or myocardial ischemia. J. Mol. Cell. Cardiol. 48, 1236-1244. doi: 10.1016/j.yjmcc.2010.02.020

Fang, X., Kaduce, T. L., Weintraub, N. L., Harmon, S., Teesch, L. M., Morisseau, C., et al. (2001). Pathways of epoxyeicosatrienoic acid metabolism in endothelial cells. Implications for the vascular effects of soluble epoxide hydrolase inhibition. J. Biol. Chem. 276, 14867-14874. doi: 10.1074/jbc.M011 761200

Feingold, K., Kim, M. S., Shigenaga, J., Moser, A., and Grunfeld, C. (2004). Altered expression of nuclear hormone receptors and coactivators in mouse heart during the acute-phase response. Am. J. Physiol. Endocrinol. Metab. 286, E201-207. doi: 10.1152/ajpendo.00205.2003

Gabay, C., and Kushner, I. (1999). Acute-phase proteins and other systemic responses to inflammation. N. Engl. J. Med. 340, 448-454. doi: 10.1056/NEJM199902113400607

Hambleton, J., Weinstein, S. L., Lem, L., and Defranco, A. L. (1996). Activation of c-Jun N-terminal kinase in bacterial lipopolysaccharide-stimulated macrophages. Proc. Natl. Acad. Sci. U.S.A. 93, 2774-2778. doi: 10.1073/pnas.93.7.2774

Imig, J. D. (2012). Epoxides and soluble epoxide hydrolase in cardiovascular physiology. Physiol. Rev. 92, 101-130. doi: 10.1152/physrev.00021.2011

Jones, D. C., Ding, X., and Daynes, R. A. (2002). Nuclear receptor peroxisome proliferator-activated receptor alpha (PPARalpha) is expressed in resting murine lymphocytes. The PPARalpha in T and B lymphocytes is both transactivation and transrepression competent. J. Biol. Chem. 277, 6838-6845. doi: 10.1074/jbc.M106908200

Katragadda, D., Batchu, S. N., Cho, W. J., Chaudhary, K. R., Falck, J. R., and Seubert, J. M. (2009). Epoxyeicosatrienoic acids limit damage to mitochondrial function following stress in cardiac cells. J. Mol. Cell. Cardiol. 46, 867-875. doi: 10.1016/j.yjmcc.2009.02.028

Kim, I. H., Morisseau, C., Watanabe, T., and Hammock, B. D. (2004). Design, synthesis, and biological activity of 1,3-disubstituted ureas as potent inhibitors of the soluble epoxide hydrolase of increased water solubility. J. Med. Chem. 47, 2110-2122. doi: 10.1021/jm030514j

Kozak, W., Aronoff, D. M., Boutaud, O., and Kozak, A. (2003). 11,12epoxyeicosatrienoic acid attenuates synthesis of prostaglandin E2 in rat monocytes stimulated with lipopolysaccharide. Exp. Biol. Med. (Maywood) 228, 786-794.

Levick, S. P., Loch, D. C., Taylor, S. M., and Janicki, J. S. (2007). Arachidonic acid metabolism as a potential mediator of cardiac fibrosis associated with inflammation. J. Immunol. 178, 641-646. doi: 10.4049/jimmunol.178.2.641

Liu, Y., Zhang, Y., Schmelzer, K., Lee, T. S., Fang, X., Zhu, Y., et al. (2005). The antiinflammatory effect of laminar flow: the role of PPARgamma, epoxyeicosatrienoic acids, and soluble epoxide hydrolase. Proc. Natl. Acad. Sci. U.S.A. 102, 16747-16752. doi: 10.1073/pnas.0508081102

Maitra, U., Chang, S., Singh, N., and Li, L. (2009). Molecular mechanism underlying the suppression of lipid oxidation during endotoxemia. Mol. Immunol. 47, 420425. doi: 10.1016/j.molimm.2009.08.023

Mishra, P., Paramasivam, S. K., Thylur, R. P., Rana, A., and Rana, B. (2010). Peroxisome proliferator-activated receptor gamma ligand-mediated apoptosis of hepatocellular carcinoma cells depends upon modulation of PI3Kinase pathway independent of Akt. J. Mol. Signal. 5, 20. doi: 10.1186/1750-2187-5-20

Moraes, L. A., Piqueras, L., and Bishop-Bailey, D. (2006). Peroxisome proliferatoractivated receptors and inflammation. Pharmacol. Ther. 110, 371-385. doi: 10.1016/j.pharmthera.2005.08.007.

Ng, V. Y., Huang, Y., Reddy, L. M., Falck, J. R., Lin, E. T., and Kroetz, D. L. (2007). Cytochrome $\mathrm{P} 450$ eicosanoids are activators of peroxisome proliferator-activated receptor alpha. Drug Metab. Dispos. 35, 1126-1134. doi: 10.1124/dmd.106. 013839

Niebauer, J., Volk, H. D., Kemp, M., Dominguez, M., Schumann, R. R., Rauchhaus, M., etal. (1999). Endotoxin and immune activation in chronic heart failure: a prospective cohort study. Lancet 353, 1838-1842. doi: 10.1016/S01406736(98)09286-9281

Node, K., Huo, Y., Ruan, X., Yang, B., Spiecker, M., Ley, K., et al. (1999). Antiinflammatory properties of cytochrome P450 epoxygenase-derived eicosanoids. Science 285, 1276-1279. doi: 10.1126/science.285.5431.1276

Oddis, C. V., and Finkel, M. S. (1995). Cytokine-stimulated nitric oxide production inhibits mitochondrial activity in cardiac myocytes. Biochem. Biophys. Res. Commun. 213, 1002-1009. doi: 10.1006/bbrc.1995.2228

Ohlsson, K., Bjork, P., Bergenfeldt, M., Hageman, R., and Thompson, R. C. (1990). Interleukin-1 receptor antagonist reduces mortality from endotoxin shock. Nature 348, 550-552. doi: 10.1038/348550a0

Opal, S. M., Scannon, P. J., Vincent, J. L., White, M., Carroll, S. F., Palardy, J. E., et al. (1999). Relationship between plasma levels of lipopolysaccharide (LPS) and LPS-binding protein in patients with severe sepsis and septic shock. J. Infect. Dis. 180, 1584-1589. doi: 10.1086/315093

Ridker, P. M., and Silvertown, J. D. (2008). Inflammation, C-reactive protein, and atherothrombosis. J. Periodontol. 79, 1544-1551. doi: 10.1902/jop.2008.080249

Roman, R. J. (2002). P-450 metabolites of arachidonic acid in the control of cardiovascular function. Physiol. Rev. 82, 131-185. doi: 10.1152/physrev.00021.2001

Rompe, F., Artuc, M., Hallberg, A., Alterman, M., Stroder, K., ThoneReineke, C., et al. (2010). Direct angiotensin II type 2 receptor stimulation acts anti-inflammatory through epoxyeicosatrienoic acid and inhibition of nuclear factor kappaB. Hypertension 55, 924-931. doi: 10.1161/HYPERTENSIONAHA.109.147843

Rosen, E. D., Sarraf, P., Troy, A. E., Bradwin, G., Moore, K., Milstone, D. S., et al. (1999). PPAR gamma is required for the differentiation of adipose tissue in vivo and in vitro. Mol. Cell. 4, 611-617. doi: 10.1016/S1097-2765(00)80211-7

Rosenthal, M. D., Rzigalinski, B. A., Blackmore, P. F., and Franson, R. C. (1995). Cellular regulation of arachidonate mobilization and metabolism. Prostaglandins Leukot. Essent. Fatty Acids. 52, 93-98. doi: 10.1016/0952-3278(95)90004-7 
Samokhvalov, V., Alsaleh, N., El-Sikhry, H. E., Jamieson, K. L., Chen, C. B., Lopaschuk, D. G., et al. (2013). Epoxyeicosatrienoic acids protect cardiac cells during starvation by modulating an autophagic response. Cell Death Dis. 4, e885. doi: $10.1038 /$ cddis. 2013.418

Samokhvalov, V., Ussher, J. R., Fillmore, N., Armstrong, I. K., Keung, W., Moroz, D., et al. (2012). Inhibition of malonyl-CoA decarboxylase reduces the inflammatory response associated with insulin resistance. Am. J. Physiol. Endocrinol. Metab. 303, E1459-E1468. doi: 10.1152/ajpendo.00018.2012

Seargent, J. M., Yates, E. A., and Gill, J. H. (2004). GW9662, a potent antagonist of PPARgamma, inhibits growth of breast tumour cells and promotes the anticancer effects of the PPARgamma agonist rosiglitazone, independently of PPARgamma activation. Br. J. Pharmacol. 143, 933-937. doi: 10.1038/sj.bjp. 0705973

Seubert, J. M., Darmon, A. J., El-Kadi, A. O., D'souza, S. J., and Bend, J. R. (2002) Apoptosis in murine hepatoma hepa 1c1c7 wild-type, C12, and C4 cells mediated by bilirubin. Mol. Pharmacol. 62, 257-264. doi: 10.1124/mol.62.2.257

Shahabi, P., Siest, G., and Visvikis-Siest, S. (2014). Influence of inflammation on cardiovascular protective effects of cytochrome P450 epoxygenasederived epoxyeicosatrienoic acids. Drug Metab. Rev. 46, 33-56. doi: 10.3109/03602532.2013.837916

Spector, A. A., and Norris, A. W. (2007). Action of epoxyeicosatrienoic acids on cellular function. Am. J. Physiol. Cell. Physiol. 292, C996-C1012. doi: 10.1152/ajpcell.00402.2006

Turdi, S., Han, X., Huff, A. F., Roe, N. D., Hu, N., Gao, F., et al. (2012). Cardiac-specific overexpression of catalase attenuates lipopolysaccharideinduced myocardial contractile dysfunction: role of autophagy. Free Radic. Biol. Med. 53, 1327-1338. doi: 10.1016/j.freeradbiomed.2012.07.084

Wang, N., Verna, L., Chen, N. G., Chen, J., Li, H., Forman, B. M., et al. (2002). Constitutive activation of peroxisome proliferator-activated receptor-gamma suppresses pro-inflammatory adhesion molecules in human vascular endothelial cells. J. Biol. Chem. 277, 34176-34181. doi: 10.1074/jbc.M203436200

Wang, P., Henning, S. M., and Heber, D. (2010). Limitations of MTT and MTS-based assays for measurement of antiproliferative activity of green tea polyphenols. PLoS ONE 5:e10202. doi: 10.1371/journal.pone.0010202

Widstrom, R. L., Norris, A. W., Van Der Veer, J., and Spector, A. A. (2003). Fatty acid-binding proteins inhibit hydration of epoxyeicosatrienoic acids by soluble epoxide hydrolase. Biochemistry 42, 11762-11767. doi: 10.1021/bi03 $4971 d$
Wray, J., and Bishop-Bailey, D. (2008). Epoxygenases and peroxisome proliferatoractivated receptors in mammalian vascular biology. Exp. Physiol. 93, 148-154 doi: 10.1113/expphysiol.2007.038612

Xu, D. Y., Davis, B. B., Wang, Z. H., Zhao, S. P., Wasti, B., Liu, Z. L., et al. (2013). A potent soluble epoxide hydrolase inhibitor, t-AUCB, acts through PPARgamma to modulate the function of endothelial progenitor cells from patients with acute myocardial infarction. Int. J. Cardiol. 167, 1298-1304. doi: 10.1016/j.ijcard.2012.03.167

Yasuda, S., and Lew, W. Y. (1997). Lipopolysaccharide depresses cardiac contractility and beta-adrenergic contractile response by decreasing myofilament response to $\mathrm{Ca}^{2+}$ in cardiac myocytes. Circ. Res. 81, 1011-1020. doi: 10.1161/01.RES.81.6.1011

Zernecke, A., Bernhagen, J., and Weber, C. (2008). Macrophage migration inhibitory factor in cardiovascular disease. Circulation 117, 1594-1602. doi: 10.1161/CIRCULATIONAHA.107.729125

Zhao, G., Wang, J., Xu, X., Jing, Y., Tu, L., Li, X., et al. (2012). Epoxyeicosatrienoic acids protect rat hearts against tumor necrosis factor-alpha-induced injury. J. Lipid Res. 53, 456-466. doi: 10.1194/jlr.M017319

Conflict of Interest Statement: The authors declare that the research was conducted in the absence of any commercial or financial relationships that could be construed as a potential conflict of interest.

Received: 15 September 2014; accepted: 23 October 2014; published online: 11 November 2014.

Citation: Samokhvalov V, Vriend J, Jamieson KL, Akhnokh MK, Manne R, Falck JR and Seubert JM (2014) PPAR $\gamma$ signaling is required for mediating EETs protective effects in neonatal cardiomyocytes exposed to LPS. Front. Pharmacol. 5:242. doi: 10.3389/fphar.2014.00242

This article was submitted to Experimental Pharmacology and Drug Discovery, a section of the journal Frontiers in Pharmacology.

Copyright (c) 2014 Samokhvalov, Vriend, Jamieson, Akhnokh, Manne, Falck and Seubert. This is an open-access article distributed under the terms of the Creative Commons Attribution License (CC BY). The use, distribution or reproduction in other forums is permitted, provided the original author(s) or licensor are credited and that the original publication in this journal is cited, in accordance with accepted academic practice. No use, distribution or reproduction is permitted which does not comply with these terms. 